\title{
Longest Survival with Renal AA Amyloidosis: Development of End Stage Renal Disease after 25 Years of AA Amyloidosis Diagnosis
}

\begin{abstract}
Keywords: AA amyloidosis; End stage renal disease; Tuberculosis Abstract

This is case report of a patient who was diagnosed with secondary AA Amyloidosis of the kidneys due to Pulmonary Tuberculosis in 1973. AA Amyloidosis was diagnosed based on renal biopsy due to heavy proteinuria. Nephrotic Syndrome and concomitant renal insufficiency resolved after treatment of tuberculosis. Patient remained proteinuria free with preservation of renal function for more than 25 years. He presented to the same hospital 25 years later with heavy proteinuria and advanced renal failure. The work up of renal failure was inconclusive during this admission. He underwent a repeat diagnostic renal biopsy which revealed AA Amyloidosis present throughout the kidney. Recta and minor Salivary gland biopsies also revealed AA amyloidosis. The patient was started on chronic renal replacement therapy but unfortunately expired after few months due to bacteremia.
\end{abstract}

\section{Introduction}

Amyloidosis is a systemic disease as a result of deposition of amyloid fibrils. The type of precursor protein, tissue distribution, and the amount of amyloid deposition define clinical manifestations of the disease. AA amyloid fibrils are derived from the acutephase reactant serum amyloid A protein (SAA) through process of cleavage, misfolding, and aggregation into a highly ordered abnormal $\beta$-sheet conformation. Amyloid fibrils associate with other moieties, including serum amyloid $\mathrm{P}$ component (SAP) resulting in deposits that disrupt the structureand function of various organs [1].

Renal amyloidosis dominates clinical presentation of systemic amyloidosis and presents with heavy proteinuria and nephrotic syndrome. AA amyloidosis can result from chronic infections like Tuberculosis and pyogenic infections [2]. Various rheumatologic diseases have been the most common cause of AA amyloidosis in Western countries accounting for $60 \%$ of all cases $[3,4]$. The deposited amyloid fibrils in the kidney may or may not undergo regression with treatment of underlying disease process [5].

The development of End Stage Renal Disease (ESRD) is variable after diagnosis of renal amyloidosis. Overall prognosis is poor with life expectancy on average of 13 months after diagnosis [6]. The survival of AA amyloidosis patients on hemodialysis is lower than that of patients with other primary renal diseases including diabetic nephropathy.

In this case report, a patient developed end stage renal disease (ESRD) after 27 years of AA amyloidosis being in remission and died after few months of initiation of chronic dialysis.

\section{Journal of}

Urology \& Nephrology

\author{
Naheed Ansari* \\ Renal Division, Department of Medicine, Jacobi Medical Center, \\ New York, USA

\section{Address for Correspondence} \\ Naheed Ansari, Renal Division, Department of Medicine, Jacobi \\ Medical Center, 1400 Pelham Parkway Bronx, New York 10461 \\ USA, E-mail: naheed.ansari@nbhn.net

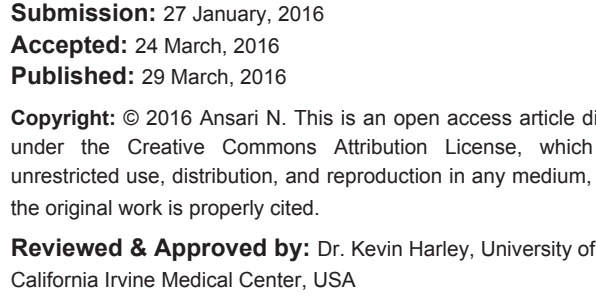

Reviewed \& Approved by: Dr. Kevin Harley, University of California Irvine Medical Center, USA

\section{Case Report}

A 69 year old man was admitted to our hospital for treatment of Pulmonary Tuberculosis (TB) in 1973. At that time he also had nephrotic syndrome for which he underwent renal biopsy which revealed Amyloidosis (hospital record documentation). He was treated with anti-TB meds which included Isoniazid and Ethambutol. Nephrotic Syndrome resolved completely and renal function remained close to baseline with treatment of TB (Figure 1).

He presented to same hospital again in 7/2000 with one month history of anorexia, weight loss, nausea and dizziness. He denied any history of Diabetes Mellitus. Physical examination revealed orthostatic changes in blood pressure measurements. No evidence of obstruction was found on post void residual measurement.

Laboratory evaluation revealed advanced renal failure with BUN/ Cr $74 / 15.4$, severe anemia with $\mathrm{H} / \mathrm{H}$ of $8 / 24$, heavy proteinuria 30 grams per day on multiple random quantifications, and severe

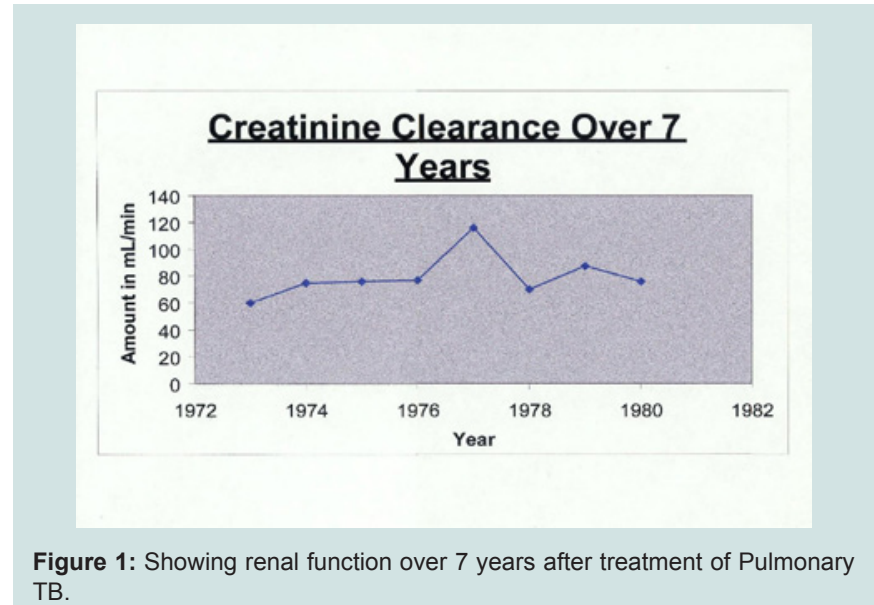


Citation: Ansari N. Longest Survival with Renal AA Amyloidosis: Development of End Stage Renal Disease after 25 Years of AA Amyloidosis Diagnosis. J Urol Nephro

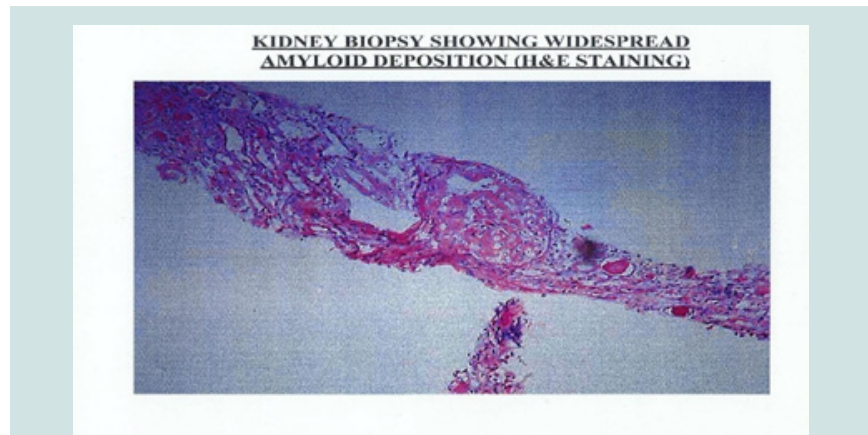

Figure 2: Showing pink amorphous material predominantly in the mesangium and glomerular capillary loops.

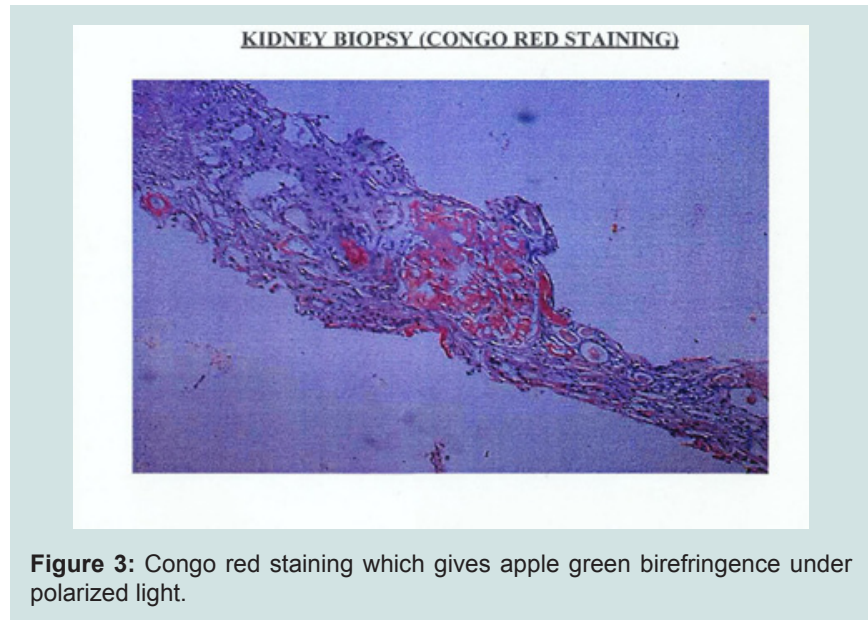

hypoalbuminemia with serum albumin level of $0.9 \mathrm{gm} / \mathrm{dl}$. Serologic work up was negative including serum immunoelectrophoresis and HIV. Work up for malignancy was negative. HbA1C value was $5.8 \%$.

Urinalysis showed fatty droplets and oval fat bodies repeatedly. Renal function did not change significantly after IV hydration. Orthostatic hypotension persisted besides massive fluid administration. Renal ultrasound showed no obstruction and Kidneys were found to be echogenic and relatively small in size ( 8.7 and 9.8 $\mathrm{cm}$ long). Chest x-ray showed no evidence of any active pulmonary disease. He underwent diagnostic renal biopsy due to persistent renal failure with heavy proteinuria. Renal biopsy revealed widespread deposition of amyloid fibrils throughout the kidney involving glomeruli, basement membranes of tubules and blood vessels walls (Figure 2). These deposits showed apple green birefringence on Congo red staining (Figure 3). Immunostaining for AA protein was used to document presence of AA fibrils (Figure 4). Staining with antibodies against kappa and lambda chains was negative which excluded AL amyloidosis. Electron microscopy revealed presence of randomly distributed throughout basement membrane consistent with amyloidosis (Figure 5). No evidence of any other renal disease was found on biopsy.

He underwent rectal and minor salivary gland biopsies which revealed deposition of AA amyloidosis throughout these two biopsy specimens (Figures 6 and 7).
There was evidence of bilateral renal vein thrombi on both Magnetic Resonance Venography (MRV) as well as renal angiography (Figures 8 and 9). These tests were performed due to high index of suspicion for bilateral renal thrombi due to severe proteinuria as possible cause of renal disease. Anticoagulant therapy with heparin and later Coumadin was initiated for bilateral renal vein thrombosis with which his renal function did not change much.

\section{Discussion}

Systemic amyloidosis is characterized by extracellular deposits on different organs of insoluble fibrils compounded of low molecular weight subunits coming from a great diversity of serum proteins. Secondary amyloidosis AA is due to fibril deposition composed of fragments of the acute phase reactant serum amyloid A (SAA). Clinical manifestations are dependent on both magnitude and site of the amyloid deposits. Time to clinical presentation is variable but progression can be fast. The diagnostic sensitivity of kidney biopsy is close to $100 \%$ in patients with renal involvement [7]

AA amyloidosis in the kidney is a rare entity. Tuberculosis used to be the commonest cause of renal AA myloidosis in the past up to

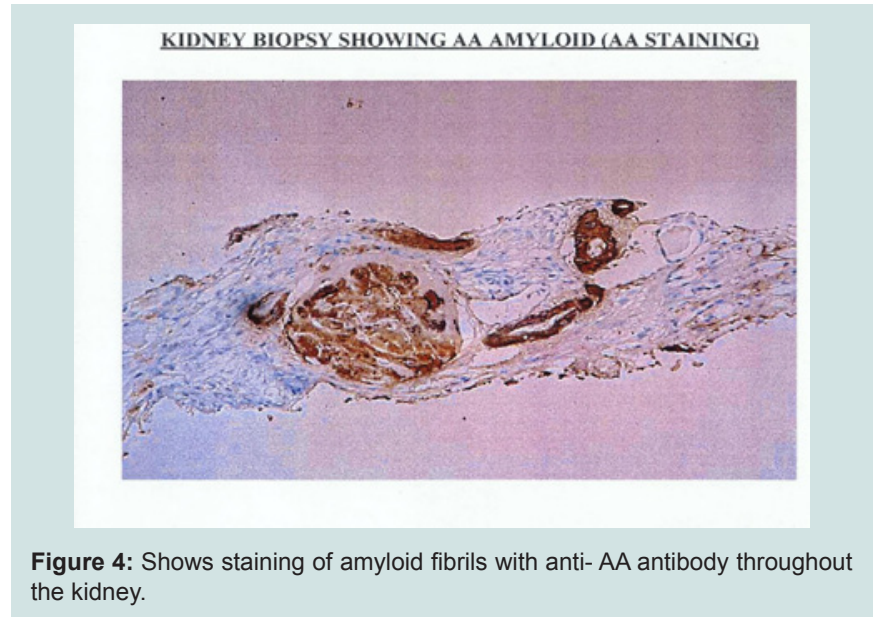

the kidney.

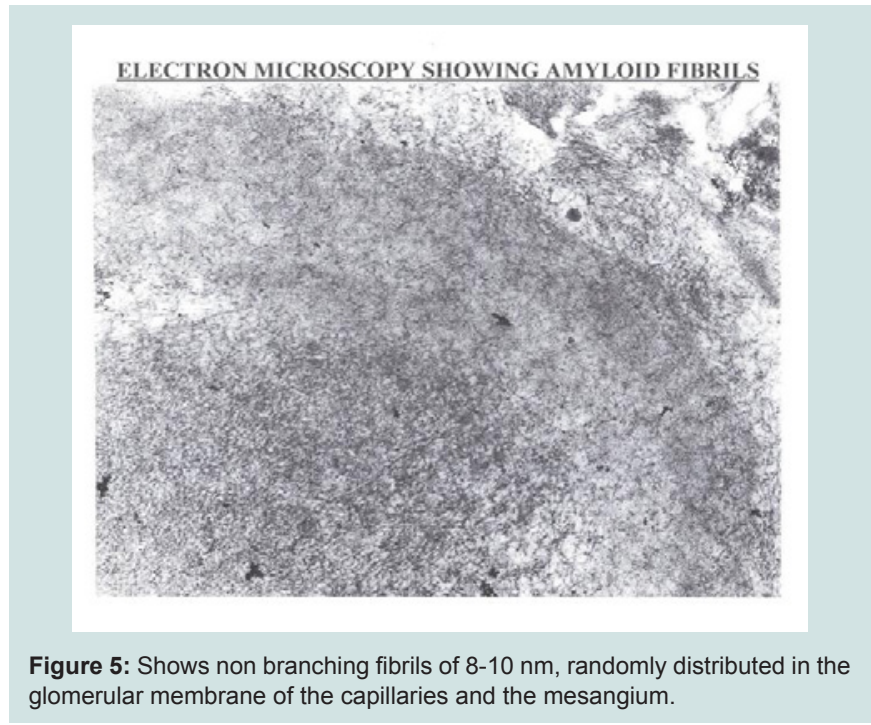


Citation: Ansari N. Longest Survival with Renal AA Amyloidosis: Development of End Stage Renal Disease after 25 Years of AA Amyloidosis Diagnosis. J Urol Nephro

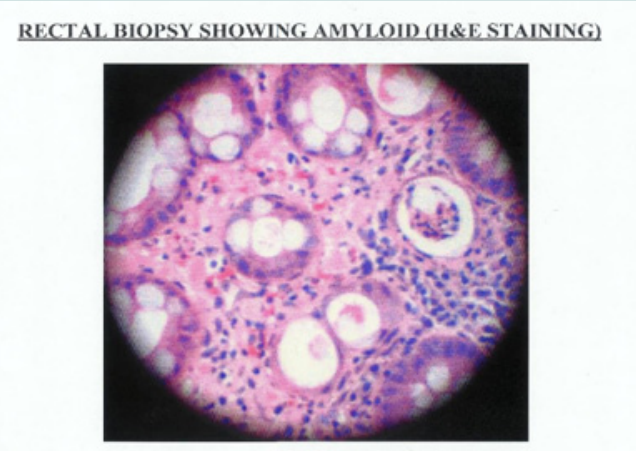

Figure 6: Shows pink amyloid material throughout the rectal tissue.

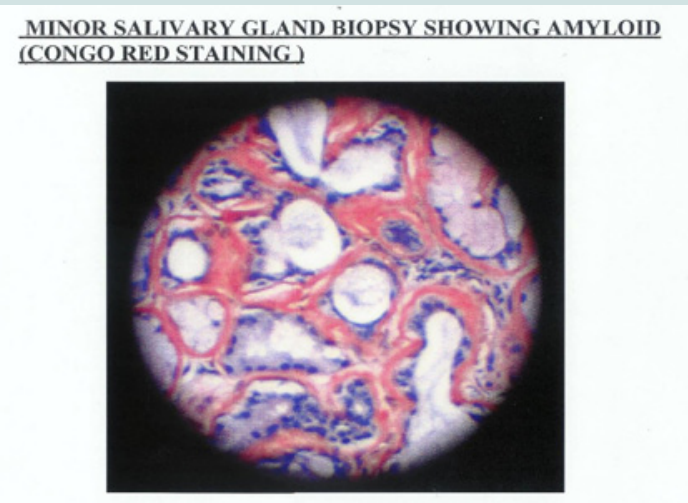

Figure 7: Showing amyloid deposition in minor salivary gland.

$50 \%[2,7]$. It is usually seen in males of $40-50$ years of age as in this patient. It has been confirmed that amyloid deposits increase rapidly in patients with a relapse of the underlying inflammatory disease. Patients with predominant glomerular deposition present usually with overt proteinuria associated with poor renal prognosis. Intensity of vascular and glomerular amyloid load along with interstitial fibrosis and tubular atrophy is shown to be correlated with poor renal outcome [8].

Serum Amyloid A Protein (SAA) is usually elevated in most cases of AA amyloidosis. In some studies, level of circulating SAA protein has been correlated with tissue amyloid load and clinical outcome in patients with AA amyloidosis $[9,10]$. A recent study on natural history of AA amyloidosis showed that patients with improvement of renal function over follow-up had an average SAA of $6 \mathrm{mg} / \mathrm{L}$ compared to $28 \mathrm{mg} / \mathrm{L}$ in patients with decline in renal function [4]. We did not follow SAP levels in this patient due to lack of availability of this test in our facility which may have helped in predicting prognosis of the disease.

Absence of edema besides severe hypoalbuminemia also remains unexplained in this patient. Formation of some intracellular osmoles to counteract effects of decreased colloid oncotic pressure due to severe hypoalbuminemia may have accounted for absence of edema in this case. Patients with HIV associated Nephropathy (HIVAN) also commonly do not manifest edema besides heavy proteinuria.

Rate of decline of renal function in renal AA amyloidosis is fast (1.07 $\mathrm{ml} / \mathrm{min} / \mathrm{month})$ as compared to other glomerular diseases [8]. The higher the albuminuria, the greater the rate of progression of renal failure ( 2.63 months vs. 62.5 months if albuminuria $>2 \mathrm{gm} / \mathrm{dl}$ ). One study has shown serum creatinine to be the strongest variable associated with poor renal survival [7]. Serum creatinine $>2 \mathrm{mg} / \mathrm{dl}$ and serum albumin value less than $2.5 \mathrm{gm} / \mathrm{dl}$ are associated with poor renal survival.

Renal vein thrombosis is an uncommon phenomenon in AA amyloidosis (3\%). Renal vein thrombosis usually seen with other common glomerular diseases like membranous Nephropathy, Minimal change disease and FSGS. The underlying pathophysiology of renal vein thrombosis is due to urinary loss of Protein C, Protein $\mathrm{S}$ and Anti-Thrombin 111 in these glomerular diseases. In AA amyloidosis, the same pathophysiology may be assumed to be the cause of thromboembolism but there are no well done studies on thromboembolism associated with renal AA amyloidosis. Whether development of bilateral renal vein thrombosis along with heavy proteinuria caused rapid deterioration of this patient's renal function remains a possibility but there was no histological evidence of renal vein thrombosis on renal biopsy and anticoagulation therapy

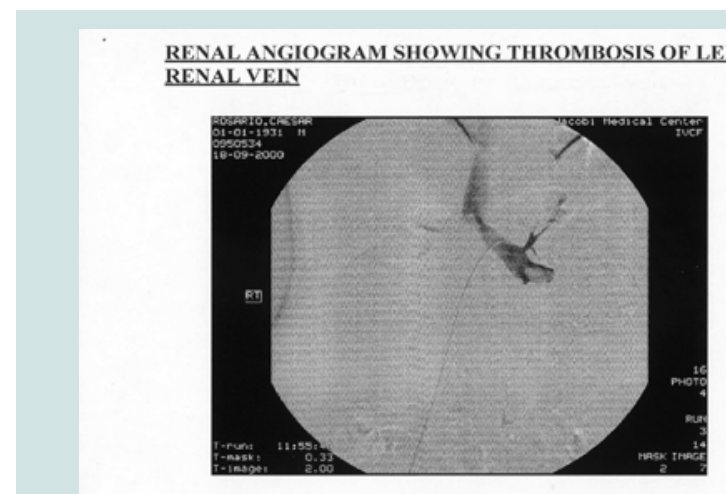

Figure 8: Showing thrombosis of left renal vein. RENAL ANGIOGRAM SHOWING THROMBOSIS OF RIGHT RENAL VEIN

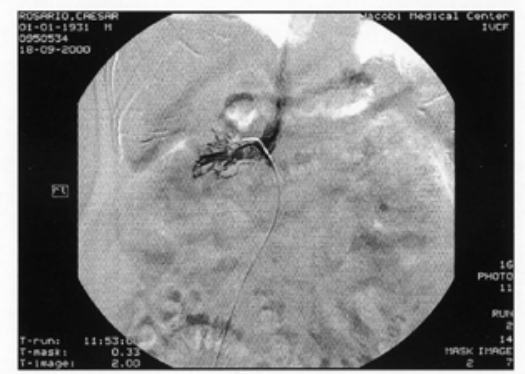

Figure 9: Showing thrombosis of right renal vein 
Citation: Ansari N. Longest Survival with Renal AA Amyloidosis: Development of End Stage Renal Disease after 25 Years of AA Amyloidosis Diagnosis. J Urol Nephro

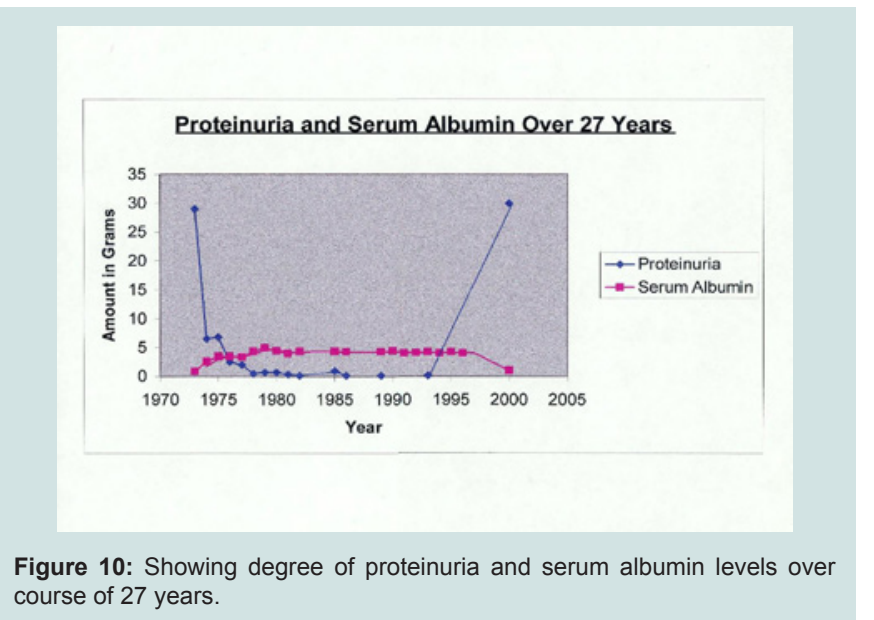

course of 27 years.

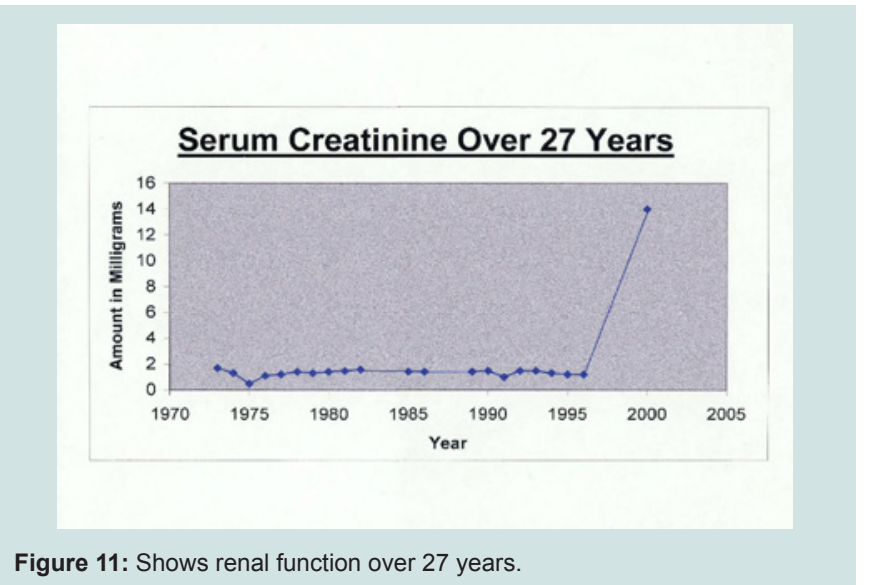

improve did not improve his renal function. However, relapse of severe proteinuria after being in remission for 25 years in absence of ongoing inflammatory process remains unexplained.

The main aim in treatment of patients with renal AA amyloidosis is to switch off production of SAA by controlling underlying disease process. The improvement in renal manifestations may be associated with regression of amyloid material in the tissues [11,12]. This can be done by using radiolabelled serum amyloid P (SAP) Scintigraphy which is available in some centers. The relationship between amyloid regression and improvement in renal function remains unclear. This patient was treated with anti-TB meds on his first presentation which resulted in resolution of Nephrotic Syndrome and stabilization of his renal function (Figures 10 and 11).

The patient was started on chronic hemodialysis which he did not tolerate very well. He passed away after few months of initiation of chronic dialysis due to sepsis.

Patients with AA amyloidosis who progress to ESRD can be treated with either dialysis or renal transplantation. Hemodialysis and continuous ambulatory peritoneal dialysis appear equally effective but extent of extrarenal amyloid deposition, hypotension with hemodialysis and peritonitis with CAPD may affect adversely effectiveness of the therapy $[13,14]$. The outcome appears to be better in patients without cardiac involvement [14]. This patient was started on hemodialysis through perm catheter which he did not tolerate very well due to persistent hypotension. The hypotension limited ultrafiltration on almost every hemodialysis session. He developed bacteremia related to dialysis catheter and did not survive besides institution of broad spectrum antibiotics and perm catheter exchange.

Renal transplantation in renal amyloidosis is mostly studied in AA amyloidosis, since patient survival in often relatively short in $\mathrm{AL}$ amyloidosis. Recently, increased survival in AL amyloidosis has been reported. This patient did not survive long enough to be referred for renal transplantation.

\section{Conclusion}

In summary, this case report shows that AA amyloidosis deposition in the kidney can be associated with complete remission of nephrotic Syndrome with treatment of underlying disease process. This is the longest survival reported so far with renal AA amyloidosis. Renal function and proteinuria can be well preserved for long time (upto 27 years) before development of ESRD. It is unclear whether these amyloid deposits in the kidney are due the same found on the first biopsy or he developed another episode of AA amyloidosis due to another inflammatory condition later in life. More studies need to be done to understand the mechanism of nephrotic syndrome and disappearance of proteinuria while amyloid deposits in the tissues may or may not undergo complete resolution.

\section{References}

1. Merlini G, Bellotti V (2003) Molecular mechanisms of amyloidosis. N Engl J Med 349: 583-596.

2. Kennedy AC, Burton JA, Allison ME (1974) Tuberculosis as a continuing cause of renal amyloidosis. Br Med J 3: 795-797.

3. Janssen S, Van Rijswijk MH, Meijer S, Ruinen L,Van der Hem GK (1986) Systemic amyloidosis: a clinical survey of 144 cases. Neth J Med 29: 376385.

4. Lachmann HJ, Goodman HJ, Gilberston JA, Gallimore JR, Sabin CA, et al. (2007) Natural history and outcome in systemic AA amyloidosis. N Engl J Med 356: 2361-2371.

5. Crowley S, Feinfeld DA, Janis R (1989) Resolution of nephrotic syndrome and lack of progression of heroin-associated renal amyloidosis. Am J Kidney Dis 13: $333-335$

6. Brunner FP, Fassbinder W, Broyer M, Oulès R, Brynger H, et al. (1988) Survival on renal replacement therapy: data from the EDTA Registry. Nephrol Dial Transplant 3: 109-122.

7. Gertz MA, Kyle RA (1991) Secondary systemic amyloidosis: response and survival in 64 patients. Medicine (Baltimore) 70: 246-256.

8. Verine J, Mourad N, Desseaux K, Vanhille P, Noël LH, et al (2007) Clinical and histological characteristics of renal $\mathrm{AA}$ amyloidosis: a retrospective study of 68 cases with a special interest to amyloid-associated inflammatory response. Hum Pathol 38: 1798-1809.

9. Gillmore JD, Lovat LB, Persey MR, Pepys MB, Hawkins PN (2001) Amyloid load and clinical outcome in $A A$ amyloidosis in relation to circulation concentration of serum amyloid A protein. Lancet 358: 24-29.

10. Joss N, McLaughlin K, Simpson K, Boulton-Jones JM (2000) Presentation, survival and prognostic markers in AA amyloidosis. QJM 93: 535-542.

11. Hawkins PN (1994) Studies with radiolabelled serum amyloid $P$ component provide evidence for turnover and regression of amyloid deposits in vivo. Clin Sci (Lond) 87: 289-295.

12. Hawkins PN (2002) Serum amyloid $P$ component scintigraphy for diagnosis and monitoring amyloidosis. Curr Opin Nephrol Hypertens 11: 649-655. 
Citation: Ansari N. Longest Survival with Renal AA Amyloidosis: Development of End Stage Renal Disease after 25 Years of AA Amyloidosis Diagnosis. J Urol Nephro

13. Ylinen K, Gröhagen-Riska C, Honkanen E, Ekstrand A, Metsärinne K, et al (1992) Outcome of patients with secondary amyloidosis in dialysis treatment. Nephrol Dial Transplant 7: 908-912.
14. Moroni G, Banfi G, Montoli A, Bucci A, Bertani T, et al. (1992) Chronic dialysis in patients with systemic amyloidosis: the experience in northern Italy. Clin Nephrol 38: 81-85.

\section{Acknowledgements}

Specials thanks to Dr. Serban Fotino who was involved in the care of this patient since 1973 and followed him up for more than 25 years. 